\title{
Formamide Dimers: A Computational and Matrix Isolation Study
}

Artur Mardyukov ${ }^{\S}$, Elsa Sánchez ${ }^{{ }^{*}}$, Pawel Rodziewicz ${ }^{\ddagger}$, Nikos L. Doltsinis ${ }^{\ddagger *}$, Wolfram Sander $^{8^{*}}$.

${ }^{\S}$ Lehrstuhl für Organische Chemie II der Ruhr-Universität Bochum, D-44780 Bochum, Germany.

‡ Lehrstuhl für Theoretische Chemie der Ruhr-Universität Bochum, D-44780 Bochum

*To whom correspondence should be addressed. 


\section{Supporting Information}

TABLE: Calculated and predicted experimental vibrational frequencies $\left(\mathrm{cm}^{-1}\right)$ of the FMA dimer $\mathbf{D}$ and $\mathbf{E} .^{a}$

MP2/cc-pVTZ

Calculated frequencies and shifts in the complex

Assignment

M calc $\begin{gathered}\text { Dimer D without } \\ \text { correction }\end{gathered}$ Dimer D corrected $\begin{aligned} & \text { Dimer E without } \\ & \text { correction }\end{aligned} \quad$ Dimer E corrected

\begin{tabular}{|c|c|c|c|c|c|c|c|c|c|}
\hline 1811.0 & $\begin{array}{l}1789.4^{\mathrm{b}} \\
1803.4^{\mathrm{c}}\end{array}$ & $\begin{array}{c}(-21.6) \\
(-7.6)\end{array}$ & $\begin{array}{l}1717.8 \\
1731.3\end{array}$ & $\begin{array}{c}(-20.7) \\
(-7.3)\end{array}$ & 1795.9 & $(-15.1)$ & 1724.1 & $(-14.5)$ & $v(\mathrm{C}=\mathrm{O})$ \\
\hline 3017.2 & $\begin{array}{l}3050.6^{b} \\
3006.9^{c}\end{array}$ & $\begin{array}{c}(+33.4) \\
(-10.3)\end{array}$ & $\begin{array}{l}2913.3 \\
2871.6\end{array}$ & $\begin{array}{c}(+31.9) \\
(-9.8)\end{array}$ & 3065.0 & $(+47.8)$ & 2927.1 & $(+45.6)$ & $v(\mathrm{C}-\mathrm{H})$ \\
\hline 3634.7 & $\begin{array}{l}3629.7^{b} \\
3495.9^{c}\end{array}$ & $\begin{array}{c}(-5) \\
(-138.8)\end{array}$ & $\begin{array}{l}3422.8 \\
3296.6\end{array}$ & $\begin{array}{c}(-4.7) \\
(-130.9)\end{array}$ & 3632.2 & $(-2.5)$ & 3425.2 & $(-2.4)$ & vs $\left(\mathrm{NH}_{2}\right)^{\mathrm{b}}$ \\
\hline 3787.1 & $\begin{array}{l}3780.3^{b} \\
3714.7^{c}\end{array}$ & $\begin{array}{l}(-6.8) \\
(-72.4)\end{array}$ & $\begin{array}{l}3542.1 \\
3480.7\end{array}$ & $\begin{array}{l}(-6.4) \\
(-67.8)\end{array}$ & 3785.6 & $(-1.5)$ & 3547.1 & $(-1.4)$ & vas $\left(\mathrm{NH}_{2}\right)^{\mathrm{C}}$ \\
\hline
\end{tabular}

${ }^{\mathrm{b}}$ FMA molecule which is out of plane in Figure 1.

${ }^{c}$ FMA molecule which is in plane in Figure 1 